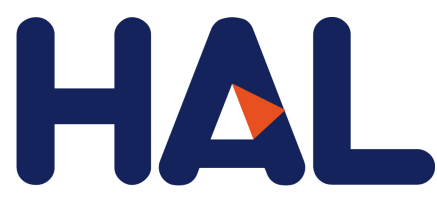

archives-ouvertes

\title{
Evaluation of the "Minimal Clinically Important Difference" (MCID) of the KOOS, KSS and SF-12 scores after open-wedge high tibial osteotomy
}

Christophe Jacquet, Charles Pioger, Raghbir Khakha, Camille Steltzlen, Kristian Kley, Nicolas Pujol, Matthieu Ollivier

\section{To cite this version:}

Christophe Jacquet, Charles Pioger, Raghbir Khakha, Camille Steltzlen, Kristian Kley, et al.. Evaluation of the "Minimal Clinically Important Difference" (MCID) of the KOOS, KSS and SF-12 scores after open-wedge high tibial osteotomy. Knee Surgery, Sports Traumatology, Arthroscopy, Springer Verlag, 2021, 29 (3), pp.820-826. 10.1007/s00167-020-06026-0 . hal-03176986

\section{HAL Id: hal-03176986 \\ https://hal.archives-ouvertes.fr/hal-03176986}

Submitted on 7 Apr 2021

HAL is a multi-disciplinary open access archive for the deposit and dissemination of scientific research documents, whether they are published or not. The documents may come from teaching and research institutions in France or abroad, or from public or private research centers.
L'archive ouverte pluridisciplinaire HAL, est destinée au dépôt et à la diffusion de documents scientifiques de niveau recherche, publiés ou non, émanant des établissements d'enseignement et de recherche français ou étrangers, des laboratoires publics ou privés. 


\title{
Evaluation of the "Minimal Clinically Important Difference" (MCID) of the KOOS, KSS and SF-12 scores after open-wedge high tibial osteotomy
}

\author{
Christophe Jacquet $^{1} \cdot$ Charles Pioger $^{2} \cdot$ Raghbir Khakha $^{1,3} \cdot$ Camille Steltzlen $^{2} \cdot$ Kristian Kley $^{1} \cdot$ Nicolas Pujol $^{2}$. \\ Matthieu Ollivier ${ }^{1}$
}

\begin{abstract}
Purpose Defining a Minimal Clinically Important Difference (MCID) value for Patient-Reported Outcome Measures (PROMs) is crucial for determining the effectiveness of a procedure and calculating the sample size for trial planning. The purpose of this study was to determine the MCID of several PROMs (Knee injury and Osteoarthritis Outcome Score (KOOS), Knee Society Score (KSS) and the SF-12) in patients who underwent medial opening-wedge High-Tibial Osteotomy (owHTO) with Patient-Specific Cutting Guides (PSCGs), using anchor-based methods.

Methods Patients undergoing isolated medial owHTO with PSCGs between January 2013 and January 2017 were enrolled in this single-center, prospective, observational study. Three outcome scores were collected pre-operatively and at the 2 years follow-up evaluation: KOOS, KSS and SF-12. The MCIDs were calculated using anchor-based method: at 2 years postoperatively: "Compared with before surgery, how would you rate operated joint now?" The responses were recorded using a five-point scale. Patients who answered "about the same" or "somewhat worse" were classified into the no change group, while those who answered "somewhat better" were classified into the minimal change group. A receiver operating characteristic (ROC) curve was used to define the cutoff point that best discriminated between the minimal change and no change groups for each PROMs

Results 196 patients were included, 75 (somewhat better) and 24 patients (about the same and somewhat worse) were, respectively, assigned to the "no change" and "minimal change" groups. There was no significant difference between the two groups in terms of baseline characteristics and postoperative complications. At 24 months follow-up all the PROMs (KOOS, KSS and SF-12) were significantly better for the "minimal change" group compared to the "no change" group. MCID was 15.4 for KOOS pain, 15.1 for KOOS symptoms, 17 for KOOS ADL, 11.2 for KOOS sports/recreation, 16.5 for KOOS QQL, 3 for KSS symptoms, 5.6 for KSS activity, 7.2 for SF-12 physical component and 6.3 for PCS mental component.

Conclusion This study determined the MCIDs of common used PROMs in patients undergoing owHTO.

Level of evidence Prospective Cohort Study, Level II
\end{abstract}

Matthieu Ollivier

matthieu.ollivier@ap-hm.fr

1 Department of Orthopedics and Traumatology, Institute of Movement and Locomotion, St. Marguerite Hospital, 270 Boulevard Sainte Marguerite, BP 29, 13274 Marseille, France

2 Département de Chirurgie orthopédique de l'hopital Mignot, Le chesnay, France

3 Guys and St Thomas' Hospital, London, UK

\section{Introduction}

In patients with moderate symptomatic tibiofemoral osteoarthritis and varus alignment of the leg, an opening-wedge High-Tibial Osteotomy (owHTO) is a joint sparing operative option $[2,3,8,18]$. Over the last decade, the outcomes have improved in owHTO by redefining the indications, improving pre-operative planning and by making the surgical technique more accurate, safer and reproducible [3, 10, 14, 27].

The use of patient-reported outcome measures (PROMs) such as Knee injury and Osteoarthritis Outcome Score 
(KOOS) [5, 26] and Knee Society Knee Score (KSS) [22], or the SF-12 [31, 32] are common and essential tools for a structured and standardized assessment of patient-perceived pain, function and life quality after owHTO surgery. There is a need of an appropriate tool to analyse the clinical relevance of the improvement in subjective outcomes after owHTO.

The Minimal Clinically Important Difference (MCID) firstly described by Jaeschle et al. [15]. as "the smallest difference in score in the domain of interest which patients perceive as beneficial and which would mandate, in the absence of troublesome side effects and excessive cost, a change in the patient's management," is a useful benchmark to determine whether patients improve enough clinically to notice a real clinical difference.

Therefore, defining a MCID value for a specific population and specific procedure is crucial for determining the effectiveness of the procedure and calculating the sample size for clinical trial planning.

To date, the MCID values have never been defined for the KOOS, KSS and SF-12 scoring system in the owHTO procedure.

The purpose of this study was to determine the MCID of several PROMs in patients who underwent owHTO with Patient Specific Cutting Guides (PSCGs), using anchorbased methods.

\section{Materials and methods}

All patients aged from 18 to 80 years old undergoing medial owHTO with PSCGs between January 2013 and January 2017 were enrolled in this single-center, prospective, observational study $(n=238)$.

Indications for owHTO included [14]: isolated medial knee osteoarthritis Ahlbäck grade $<3$ [1] with a preserved status of the patellofemoral and lateral tibio-femoral joints assessed using clinical and radiological (x-rays and MRIscan) methods, a significant metaphyseal tibial varus [Medial plateau tibial angle (MPTA) $<84^{\circ}$ ], a stable knee in sagittal and coronal plane and the failure of all non-surgical treatments.

Absolute contraindications included inflammatory joint disease, unresolved or recent septic arthritis, or deficient soft-tissue coverage.

Exclusion criteria for the present study included: concomitant cartilage surgery $(n=8)$ and preoperatively planned modification of the angulation of the tibial slope $(n=11)$.

All the surgeries were performed by two senior surgeons in a single center.

In the preoperative planning stage, the planned correction was first calculated by the surgeon using conventional radiographs (weight bearing long-leg, $\mathrm{A} / \mathrm{P}$ and lateral views) following Miniaci's Method [9]. Subsequently all patients underwent a CT-scan and a Patient-Specific Cutting Guide was designed to achieve the osteotomy.

\section{Surgical technique [3]}

After performing the standard medial surgical approach, a PSCG was used following the technique described by Chaouche et al. [3]. The plate (Activmotion ${ }^{\circledR}$, Newclip Technics, Haute-Goulaine, France) was secured using six locking screws. The osteotomy gap was filled with femoral head allograft.

The postoperative rehabilitation protocol has been previously described [10] with full weight bearing and unlimited range of motion as tolerated. The patient was advanced to full weight bearing, closed chain strengthening, and cycling by 3 weeks. Progression to sport-specific activities and full training was permitted by 12 weeks post- operatively.

In case of Lateral Hinge fractures (LHF) [30]:

For Takeuchi Type 1 and 2 diagnosed intraoperatively; toe touch weight bearing for 6 weeks with the aid of crutches was recommended. Full weight bearing was allowed after 6 weeks.

For Takeuchi 3 diagnosed intraoperatively: the fracture was treated intraoperatively by two additional compression screw and non-weight bearing for 6 weeks followed by toe touch weight bearing for 6 weeks with the aid of crutches was recommended. Full weight bearing was allowed after 12 weeks.

If a hinge fracture was diagnosed at 6 weeks' followup, for the type 1 and 2, toe touch weight bearing was not extended. For the type 3 a toe touch weight bearing for 6 weeks was recommended before full weight bearing.

\section{Patients' evaluation and follow-up}

Patients were prospectively evaluated at months $1,3,6,12$, 18 and 24 months follow-up. Radiographic outcomes were evaluated with intra- and postoperative fluoroscopic imaging and with CT imaging at 6 weeks post owHTO surgery. Every radiograph and CT-scan was reviewed by two senior clinicians (one radiologist and one surgeon). In instances of different interpretation, a third evaluation was performed by the two clinicians to reach a definitive interpretation. The Kappa coefficient of the first evaluation was 0.95. Lateral hinge fractures were classified following the Takeuchi classification [30].

Postoperative infections and bone union were also evaluated during the survey. With regards to postoperative infections, superficial infections requiring only antibiotic therapy were separated from the deep infections treated by a surgical debridement associated with antibiotic therapy.

A total of three outcome scores were collected pre-operatively and at the 2 years follow-up evaluation: Knee Society 
Knee Score (KSS), SF-12 Score, and Knee injury and Osteoarthritis Score (KOOS).

KOOS comprises a 42-item self-reported questionnaire of subscales that include pain (nine items), other symptoms (seven items), function in daily living (17 items), function in sport and recreation (five items), and knee-related quality of life (QOL) (four items), which are scored individually from 0 (extreme knee problems) to 100 (no knee problems).

The SF-12 score, which is derived from the SF-36 score, comprises a 12-item questionnaire evaluating specific factors of general health-related QOL that are divided into the physical (PCS) and mental (MCS) component summaries. The mean score of the general population is 50, with an SD of 10. Higher scores demonstrate better health-related QOL.

The KSS was developed and published in 2012 and has been validated to better characterize the expectations, satisfaction, and physical activities of the younger and more diverse population of patients undergoing total knee arthroplasty [28]. The internal properties and responses of each patient can be captured in every domain of the subjective score, including symptoms (/25), satisfaction (/40), and expectation (/15). Various kind of activities (/100), including essential activities, higher-level activities, and discretionary/ recreational activities are also evaluated by KSS [22]. For this study, two sub-scales were included: KSS symptoms (/25) and KSS activity (/100). Indeed, the sub-scale KSS satisfaction items are similar to those of the anchor method and could introduce a bias in the case of discordant results. For the sub-scale KSS expectation, it is not possible to perform a MCID estimation since the pre-operative questionnaire for expectation is different from that used post-operatively.
Patients without a 2-year follow-up evaluation were excluded from the analysis $(n=23 ; 10.5 \%)$ (Fig. 1 : Flow Chart).

\section{Calculation of minimal clinically important differences}

The MCIDs were calculated using four anchor-based methods [17]. Patients were given the anchor question at 2 years postoperatively: "Compared with before surgery, how would you rate each operated joint now?" The responses were recorded using a 5-point scale: "much better," "somewhat better," "about the same," "somewhat worse," and "much worse." Patients who answered "about the same" or "somewhat worse" were classified into the no change group, while those who answered "somewhat better" were classified into the minimal change group. Patients who answered "much better" or "much worse" were not included in the analysis because they experienced more than minimal change. Four anchor-based methods were used to calculate the MCID. The average change corresponded to the mean change in the score of the minimal change group. The minimum detectable change (MDC) approach defines minimal change as the smallest change that can be considered above the measurement error with a given level of confidence (95\%). Therefore, the MCID is equal to the upper value of the $95 \%$ confidence interval for the average change in score that is seen in the no change group. The difference in change was defined as the difference in the average change in score between the minimal change and no change groups. A receiver operating characteristic (ROC) curve was used to define the cutoff

Fig. 1 Flow chart

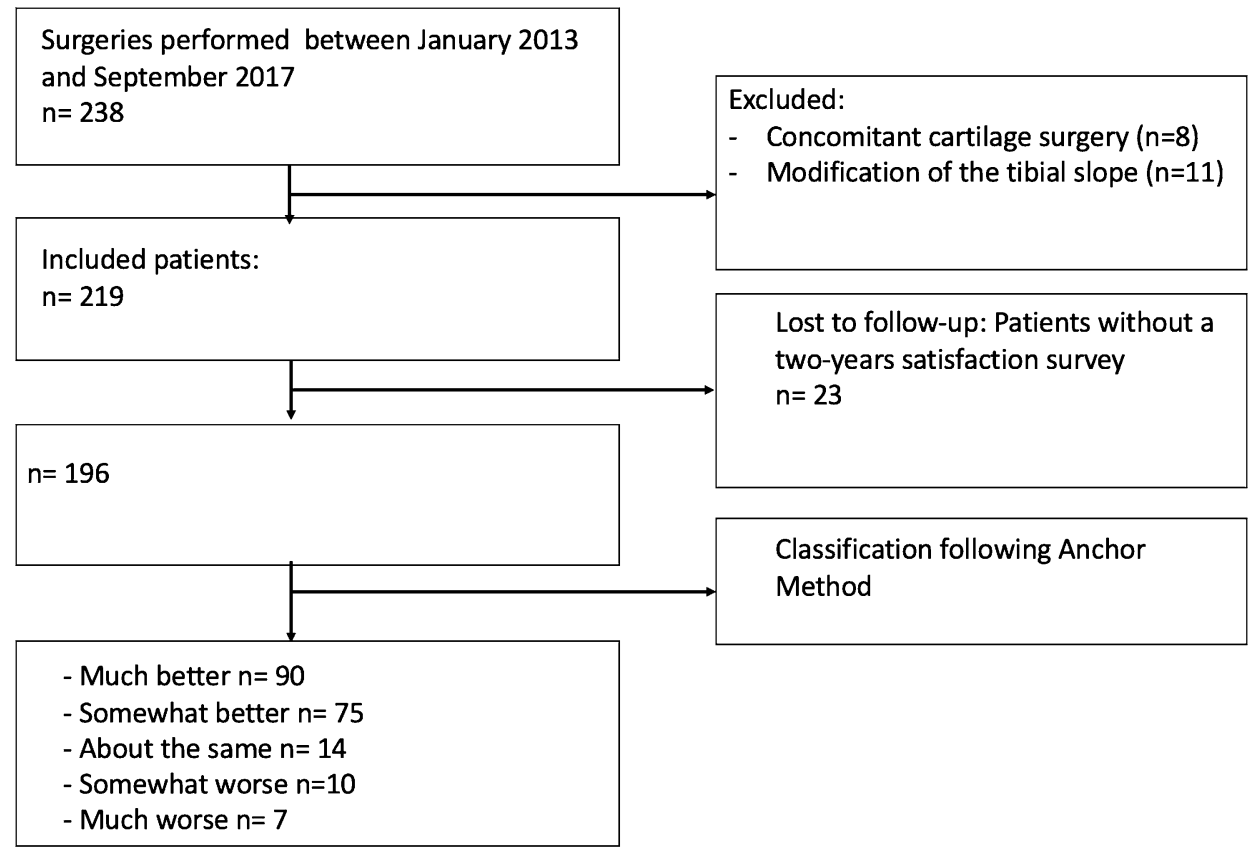


point that best discriminated between the minimal change and no change groups. The optimal cutoff point was estimated using the point that maximized both specificity and sensitivity. The Area Under the ROC Curve (AUC) was calculated to assess reliability. An AUC value of $0.7-0.8$ was considered acceptable and an AUC value of 0.8 to 0.9 was considered excellent [6].

Local Ethical Committee approval was obtained prior to study's initiation [Comité Informatique et Liberté (CIL)/ Assistance Publique des Hopitaux de Marseille (AP-HM)/ Registration Number 2019-17-129)].

\section{Statistical analysis}

The descriptive statistics were presented as mean \pm standard deviation for quantitative variables. Normal (Gaussian) distributions were verified to determine adequate statistical testing method (either parametric or non-parametric) to estimate the difference between groups in an univariate analysis.

Mann-Whitney $U$ test for two independent samples with 95\% confidence interval $(95 \% \mathrm{CI})$ was used to evaluate the differences between two variables, one-way ANOVA between more than two variables and multiple linear regressions for relationships. Paired student's $t$ test was used to estimate the evolution of functional outcomes during followup. The differences in proportions between the two samples were estimated with $z$ test with 95\% CI. PASW Statistics version 20 (SPSS, IBM Inc., Chicago, Illinois) was used for statistical analysis. The threshold for statistical significance was set at $p<0.05$.

\section{Results}

One hundred and ninety-six patients were evaluated at 2-years post-operatively and finally included in this study. A significant improvement was observed for all the cohort between the preoperative functional scores (KOOS, KSS and SF-12) and the 24 months postoperative functional scores (Table 1).

Two non-unions were seen at 9 months and 10 months after owHTO, union was achieved at 5 and 7 months, respectively, after a revision procedure. No patients had owHTO conversion to knee arthroplasty during the 2-years follow-up.

Ninety $(45,9 \%)$ patients responded that their knees were "much better" than they were before surgery, 75 (38.3\%) patients responded that their knees were "somewhat better", $14(7.1 \%)$ responded that they were"about the same", ten $(5.1 \%)$ reported that they were "somewhat worse", and seven $(3.6 \%)$ reported that their knees were "much worse". 75 (somewhat better) and 24 patients (about the same and somewhat worse) were, respectively, assigned to the
Table 1 Pre- and postoperative (24 months follow-up) PROMs of the 196 patients

\begin{tabular}{llll}
\hline PROMS & Pre-operative & 24 months & $p$ \\
\hline KOOS & & & \\
Pain & $51.4 \pm 17.9$ & $90.3 \pm 13.7$ & $<0.001$ \\
Symptoms & $45.5 \pm 19.6$ & $86.5 \pm 14.4$ & $<0.001$ \\
ADL & $45.0 \pm 18.5$ & $83.8 \pm 18.8$ & $<0.001$ \\
Sport/rec & $34.2 \pm 20.1$ & $75.6 \pm 24.2$ & $<0.001$ \\
QQL & $41.2 \pm 13.9$ & $80.7 \pm 23.1$ & \\
KSS & & & \\
Symptoms & $15.0 \pm 4.1$ & $19.4 \pm 5.1$ & 0.002 \\
Activity & $49.5 \pm 17.0$ & $82.3 \pm 13.3$ & 0.03 \\
SF-12 & & & $<0.001$ \\
PCS & $30.1 \pm 11.3$ & $49.3 \pm 11.7$ & $<0.001$ \\
MCS & $40.3 \pm 10.4$ & $54.4 \pm 10.3$ & \\
\hline
\end{tabular}

PROMs patient-reported outcome measures, KOOS Knee Injury and Osteoarthritis Outcome Score, $A D L$ activities of daily living, $Q O L$ quality of life, KSS Knee society Score, SF-12 Short Form-12, PCS Physical Component Summary, MCS Mental Component Summary

Table 2 Patient's characteristics and post-operative complications following Anchor distribution

\begin{tabular}{llll}
\hline Parameters & $\begin{array}{l}\text { No change } \\
\text { group } \\
(n=24)\end{array}$ & $\begin{array}{l}\text { Minimal } \\
\text { change group } \\
(n=75)\end{array}$ & $p$ \\
\hline Mean age (years) & $47 \pm 5$ & $49 \pm 6$ & $\mathrm{n} . \mathrm{s}$ \\
Mean BMI $\left(\mathrm{Kg} / \mathrm{m}^{2}\right)$ & $26.6 \pm 2.8$ & $27.1 \pm 3.1$ & $\mathrm{n} . \mathrm{s}$ \\
Gender (\% of men) & 67 & 62 & $\mathrm{n} . \mathrm{s}$ \\
Labor load & & & \\
Sitting $(\%)$ & 37 & 31 & $\mathrm{n} . \mathrm{s}$ \\
Standing without labor $(\%)$ & 11 & 14 & $\mathrm{n} . \mathrm{s}$ \\
Standing with labor $(\%)$ & 52 & 55 & $\mathrm{n} . \mathrm{s}$ \\
Mean pre-operative HKA $\left(^{\circ}\right)$ & $171.5 \pm 4.2$ & $172.3 \pm 5.1$ & $\mathrm{n} . \mathrm{s}$ \\
Mean Post-operative HKA $\left({ }^{\circ}\right)$ & $180.3 \pm 2.4$ & $180.7 \pm 1.9$ & $\mathrm{n} . \mathrm{s}$ \\
Lateral hinge fracture & $4(16.7 \%)$ & $11(14.7 \%)$ & $\mathrm{n} . \mathrm{s}$ \\
Type 1 $(n)$ & $3(12.5 \%)$ & $8(10.7 \%)$ & \\
Type 2 $(n)$ & $1(4.2 \%)$ & $2(2.7 \%)$ & $\mathrm{n} / \mathrm{a}$ \\
Type 3 $(n)$ & $0(0 \%)$ & $1(1.3 \%)$ & \\
Infections & & & $\mathrm{n} / \mathrm{a}$ \\
Superficial $(n)$ & 1 & 0 & $\mathrm{n} / \mathrm{a}$ \\
Deep $(n)$ & 0 & 0 & $\mathrm{n} / \mathrm{a}$ \\
Bone nonunion $(n)$ & 0 & 0 & \\
Hardware removal $(n)$ & $5(20.8 \%)$ & $18(24 \%)$ & $\mathrm{n}$ \\
\hline
\end{tabular}

$H K A$ hip-knee-ankle angle

"no change" and "minimal change" groups for the MCID calculation.

There was no significant difference between these two groups in terms of baseline characteristics and postoperative complications (Table 2). 
Table 3 Delta (pre-operative-24 months postoperative) PROMs following Anchor classification

\begin{tabular}{lllc}
\hline PROMS & No change $(n=24)$ & $\begin{array}{l}\text { Minimal change } p \\
(n=75)\end{array}$ \\
\hline KOOS & & & \\
Pain & $8.0 \pm 3.6$ & $23.3 \pm 2.0$ & 0.03 \\
Symptoms & $9.0 \pm 2.1$ & $24.1 \pm 3.5$ & 0.04 \\
ADL & $8.9 \pm 2.5$ & $21.3 \pm 4.7$ & 0.04 \\
Sport/rec & $4.5 \pm 3.9$ & $24.8 \pm 5.4$ & $<0.001$ \\
QQL & $9.4 \pm 2.3$ & $20.7 \pm 3.9$ & 0.04 \\
KSS & & & \\
Symptoms & $2.3 \pm 0.9$ & $6.3 \pm 1.1$ & $<0.001$ \\
Activity & $5.2 \pm 1.8$ & $29.4 \pm 2.1$ & $<0.001$ \\
SF-12 & & & \\
PCS & $4.8 \pm 2.2$ & $13.7 \pm 3.4$ & 0.01 \\
MCS & $4.3 \pm 1.9$ & $11.1 \pm 2.9$ & 0.03 \\
\hline
\end{tabular}

PROMs patient-reported outcome measures, KOOS Knee Injury and Osteoarthritis Outcome Score, $A D L$ activities of daily living, $Q O L$ quality of life, KSS Knee society Score, SF-12 Short Form-12, PCS Physical Component Summary, MCS Mental Component Summary, $M D C$ Minimal Detectable Change

Table 4 MCID values for the PROMs

\begin{tabular}{llllll}
\hline PROMS & Average change & $\begin{array}{l}\text { Change } \\
\text { difference }\end{array}$ & MDC & MCID & AUC \\
\hline KOOS & & & & & \\
Pain & 23.3 & 15.3 & 3.6 & 15.4 & 0.77 \\
Symptoms & 24.1 & 15.1 & 2.9 & 15.1 & 0.76 \\
ADL & 21.3 & 12.4 & 3.9 & 17.0 & 0.73 \\
Sport/rec & 24.8 & 20.3 & 3.7 & 11.2 & 0.78 \\
QQL & 20.7 & 11.3 & 3.4 & 16.5 & 0.71 \\
KSS & & & & & \\
Symptoms & 6.3 & 4.0 & 0.4 & 3.0 & 0.89 \\
Activity & 29.4 & 24.2 & 0.8 & 5.6 & 0.91 \\
SF-12 & & & & & \\
PCS & 13.7 & 8.9 & 2.1 & 7.2 & 0.78 \\
MCS & 11.1 & 6.8 & 1.3 & 6.3 & 0.74 \\
\hline
\end{tabular}

PROMs patient-reported outcome measures, KOOS Knee Injury and Osteoarthritis Outcome Score, $A D L$ activities of daily living, $Q O L$ quality of life, KSS Knee society Score, $S F-12$ Short Form-12, PCS Physical Component Summary, MCS Mental Component Summary, $M D C$ Minimal Detectable Change, $M C I D$ Minimal Clinically Important Difference using the ROC curve Anchor method, $A U C$ area Under the ROC Curve

At 24 months follow-up all the PROMs (KOOS, KSS and SF-12) were significantly better for the "minimal change" group compared to the "no change" group (Table 3).

The MCIDs for each PROM calculated with the four anchor-based methods were presented in Table 4 .
The AUC defined by the ROC were considered acceptable $(>0.7,<0.8)$ for the all the subscale of the KOOS and SF-12 score and excellent $(>0.8)$ for the two subscales of the KSS.

\section{Discussion}

The most important finding of this study was the identification of the MCID regarding the KOOS, KSS and SF12 scores in patients undergoing owHTO for primary and isolated medial knee osteoarthritis (OA). For this procedure, MCID values were 15.4 for KOOS pain, 15.1 for KOOS symptoms, 17 for KOOS ADL, 11.2 for KOOS sports/recreation, 16.5 for KOOS QQL, 3 for KSS symptoms, 5.6 for KSS activity, 7.2 for SF-12 physical component and 6.3 for PCS mental component.

Although some of the literature reporting on outcomes after owHTO estimated long-term clinical success as it relates to HTO survivorship, with failure defined by the need for revision procedure or conversion to total knee replacement $[7,11,16,24]$ much of publications focus on short and mid-term outcomes through knee-specific PROMs, without providing MCIDs [4, 12, 20, 29]. Yet, the target population is most often young (less than 60 years-old), able to deal with pain and to be more likely to prefer non-operative option [13]. Consequently, it is useful to draw upon reliable definitions of clinically meaningful improvement after owHTO.

While it is common to provide a statistically significant difference in subjective knee scores, it can be more difficult to assess the clinical meaning of this difference for the patient. Therefore, the MCID determination is relevant in the analysis of the literature's outcomes, especially since taking into account patient perspectives is more widely established, allowing to standardize this type of statistical instrument [21, 23]. Moreover, information about MCID is helpful to calculate power analysis and determine the number of subjects required.

While there is a high prevalence of MCID investigations after total knee arthroplasty [21], the present study is the only one to have investigated MCID for subjective outcomes after owHTO. Even if no consensus has been reached on the approach used to quantify MCID [19] the four anchor-based method remains the most reliable one as it involves patients [25]. The current study used the same methodology as other recent studies [23] which have already highlighted its effectiveness as a key instrument for MCID estimation. The other strengths were a large cohort with almost 200 patients enrolled prospectively and operated in a single-center, with the same selection, surgical procedure and postoperative rehabilitation.

However, there are several limitations to this study. First, the anchor-based method, despite its advantages, can lead 
to a memory bias. Second, some patients' characteristics that might affect survey responses have not been taken into account, such as gender, body mass index (BMI), comorbidity and mental health status. Finally, MCID values quantified in this study can only be applied to cases of owHTO performed in primary and isolated medial OA and in a stable knee.

\section{Conclusion}

In conclusion, our study successfully quantified the MCIDs of three frequently used PROMs such as KOOS, SF-12 and KSS scores after OWHTO. Moreover, these MCID values can be helpful to guide clinical decision-making for surgeons considering OWHTO and to handle preoperative patient expectations.

Author contributions $\mathrm{CJ}$ and $\mathrm{CP}$ have made substantial contributions to acquisition of data, or analysis and interpretation of data. NP, CS, $\mathrm{RK}$ and KK have been involved in drafting the manuscript or revising it critically. MO has made substantial contributions to conception and design, or acquisition of data, or analysis and interpretation of data. Every authors have given final approval of the version to be published and agree to be accountable for all aspects of the work in ensuring that questions related to the accuracy or integrity of any part of the work are appropriately investigated and resolved.

Funding No funding was needed for this study.

\section{Compliance with ethical standards}

Conflict of interest $\mathrm{CP}, \mathrm{CJ}$ and RK have nothing to disclose. MO and $\mathrm{KK}$ are educational consultant for Stryker, arthrex and newclip. NP and CS is educational consultant for Zimmer/Biomet and Smith \& Nephew.

Ethical approval The local ethics committee approved our study protocol prior to investigation.

\section{References}

1. Ahlbäck S, Rydberg J (1980) X-ray classification and examination technics in gonarthrosis. Lakartidningen 77(2091-2093):2096

2. Brouwer RW, Bierma-Zeinstra SMA, van Raaij TM, Verhaar JAN (2006) Osteotomy for medial compartment arthritis of the knee using a closing wedge or an opening wedge controlled by a Puddu plate. A one-year randomised, controlled study. J Bone Joint Surg Br 88:1454-1459

3. Chaouche S, Jacquet C, Fabre-Aubrespy M, Sharma A, Argenson J-N, Parratte S, Ollivier M (2019) Patient-specific cutting guides for open-wedge high tibial osteotomy: safety and accuracy analysis of a hundred patients continuous cohort. Int Orthop 43:2757-2765

4. Cho W-J, Kim J-M, Kim W-K, Kim D-E, Kim N-K, Bin S-I (2018) Mobile-bearing unicompartmental knee arthroplasty in old-aged patients demonstrates superior short-term clinical outcomes to open-wedge high tibial osteotomy in middle-aged patients with advanced isolated medial osteoarthritis. Int Orthop 42:2357-2363

5. Collins NJ, Prinsen CAC, Christensen R, Bartels EM, Terwee CB, Roos EM (2016) Knee Injury and Osteoarthritis Outcome Score (KOOS): systematic review and meta-analysis of measurement properties. Osteoarthr Cartil 24:1317-1329

6. Copay AG, Subach BR, Glassman SD, Polly DW, Schuler TC (2007) Understanding the minimum clinically important difference: a review of concepts and methods. Spine J 7:541-546

7. Darees M, Putman S, Brosset T, Roumazeille T, Pasquier G, Migaud H (2018) Opening-wedge high tibial osteotomy performed with locking plate fixation (TomoFix) and early weightbearing but without filling the defect. A concise follow-up note of 48 cases at 10 years' follow-up. Orthop Traumatol Surg Res 104:477-480

8. Duivenvoorden T, Brouwer RW, Baan A, Bos PK, Reijman M, Bierma-Zeinstra SMA, Verhaar JAN, (2014) Comparison of closing-wedge and opening-wedge high tibial osteotomy for medial compartment osteoarthritis of the knee: a randomized controlled trial with a six-year follow-up. J Bone Joint Surg Am 96:1425-1432

9. Elson DW, Petheram TG, Dawson MJ (2015) High reliability in digital planning of medial opening wedge high tibial osteotomy, using Miniaci's method. Knee Surg Sports Traumatol Arthrosc 23:2041-2048

10. Gulagaci F, Jacquet C, Ehlinger M, Sharma A, Kley K, Wilson A, Parratte S, Ollivier M (2019) A protective hinge wire, intersecting the osteotomy plane, can reduce the occurrence of perioperative hinge fractures in medial opening wedge osteotomy. Knee Surg Sports Traumatol Arthrosc. https://doi.org/10.1007/s00167-01905806-7

11. Han JH, Yang J-H, Bhandare NN, Suh DW, Lee JS, Chang YS, Yeom JW, Nha KW (2016) Total knee arthroplasty after failed high tibial osteotomy: a systematic review of open versus closed wedge osteotomy. Knee Surg Sports Traumatol Arthrosc 24:2567-2577

12. Hantes ME, Natsaridis P, Koutalos AA, Ono Y, Doxariotis N, Malizos KN (2017) Satisfactory functional and radiological outcomes can be expected in young patients under 45 years old after open wedge high tibial osteotomy in a long-term follow-up. Knee Surg Sports Traumatol Arthrosc 26:3199-3205

13. Hermann W, Lambova S, Muller-Ladner U (2018) Current treatment options for osteoarthritis. Curr Rheumatol Rev 14:108-116

14. Jacquet C, Sharma A, Fabre M, Ehlinger M, Argenson J-N, Parratte S, Ollivier M (2019) Patient-specific high-tibial osteotomy's "cutting-guides" decrease operating time and the number of fluoroscopic images taken after a Brief Learning Curve. Knee Surg Sports Traumatol Arthrosc. https://doi.org/10.1007/s00167-01905637-6

15. Jaeschke R, Singer J, Guyatt GH (1989) Measurement of health status. Ascertaining the minimal clinically important difference. Control Clin Trials 10:407-415

16. Jin C, Song E-K, Santoso A, Ingale PS, Choi I-S, Seon J-K (2019) Survival and risk factor analysis of medial open wedge high tibial osteotomy for unicompartment knee osteoarthritis. J Arthrosc Relat Surg 36:535-543

17. Katz NP, Paillard FC, Ekman E (2015) Determining the clinical importance of treatment benefits for interventions for painful orthopedic conditions. J Orthop Surg 10:24

18. Lind-Hansen TB, Lind MC, Nielsen PT, Laursen MB (2016) Open-wedge high tibial osteotomy: RCT 2 years RSA follow-up. J Knee Surg 29:664-672

19. Lizaur-Utrilla A, Gonzalez-Parreño S, Martinez-Mendez D, Miralles-Muñoz FA, Lopez-Prats FA (2019) Minimal clinically important differences and substantial clinical benefits for Knee 
Society Scores. Knee Surg Sports Traumatol Arthrosc. https:// doi.org/10.1007/s00167-019-05543-x

20. Lu J, Tang S, Wang Y, Li Y, Liu C, Niu Y, Cao P, Wang F (2019) Clinical outcomes of closing- and opening-wedge high tibial osteotomy for treatment of anteromedial unicompartmental knee osteoarthritis. J Knee Surg 32:758-763

21. Maltenfort M, Díaz-Ledezma C (2017) statistics in brief: minimum clinically important difference-availability of reliable estimates. Clin Orthop 475:933-946

22. Noble PC, Scuderi GR, Brekke AC, Sikorskii A, Benjamin JB, Lonner JH, Chadha P, Daylamani DA, Scott WN, Bourne RB (2012) Development of a new Knee Society scoring system. Clin Orthop 470:20-32

23. Ogura T, Ackermann J, Mestriner AB, Merkely G, Gomoll AH (2018) The minimal clinically important difference and substantial clinical benefit in the patient-reported outcome measures of patients undergoing osteochondral allograft transplantation in the knee. Cartilage 19:47603518812552

24. Pannell WC, Heidari KS, Mayer EN, Zimmerman K, Heckmann N, McKnight B, Hill JR, Vangsness CT, Hatch GF, Weber AE (2019) High tibial osteotomy survivorship: a population-based study. Orthop J Sports Med 7:2325967119890693

25. Revicki D, Hays RD, Cella D, Sloan J (2008) Recommended methods for determining responsiveness and minimally important differences for patient-reported outcomes. J Clin Epidemiol 61:102-109

26. Roos EM, Lohmander LS (2003) The Knee injury and Osteoarthritis Outcome Score (KOOS): from joint injury to osteoarthritis. Health Qual Life Outcomes 1:64
27. Saragaglia D, Roberts J (2005) Navigated osteotomies around the knee in 170 patients with osteoarthritis secondary to genu varum. Orthopedics 28:s1269-1274

28. Scuderi GR, Bourne RB, Noble PC, Benjamin JB, Lonner JH, Scott WN (2012) The new knee society knee scoring system. Clin Orthop 470:3-19

29. Seo S-S, Kim O-G, Seo J-H, Kim D-H, Kim Y-G, Lee I-S (2016) Complications and short-term outcomes of medial opening wedge high tibial osteotomy using a locking plate for medial osteoarthritis of the knee. Knee Surg Relat Res 28:289-296

30. Takeuchi R, Ishikawa H, Kumagai K, Yamaguchi Y, Chiba N, Akamatsu Y, Saito T (2012) Fractures around the lateral cortical hinge after a medial opening-wedge high tibial osteotomy: a new classification of lateral hinge fracture. J Arthrosc Relat Surg 28:85-94

31. Ware J, Kosinski M, Keller SD (1996) A 12-Item Short-Form Health Survey: construction of scales and preliminary tests of reliability and validity. Med Care 34:220-233

32. Webster KE, Feller JA (2016) Comparison of the short form-12 (SF-12) health status questionnaire with the SF-36 in patients with knee osteoarthritis who have replacement surgery. Knee Surg Sports Traumatol Arthrosc 24:2620-2626 